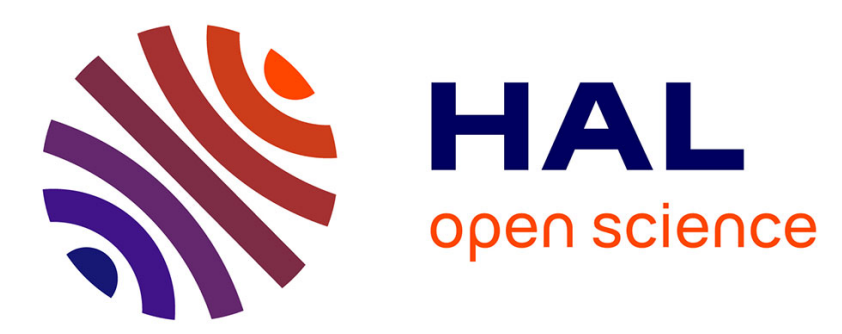

\title{
Inverse Patch Transfer Functions Method as a Tool for Source Field Identification
}

Dorian Vigoureux, Nicolas Totaro, Jonathan Lagneaux, Jean-Louis Guyader

\section{To cite this version:}

Dorian Vigoureux, Nicolas Totaro, Jonathan Lagneaux, Jean-Louis Guyader. Inverse Patch Transfer Functions Method as a Tool for Source Field Identification. Journal of Vibration and Acoustics, 2015, 137 (2), pp.021006. 10.1115/1.4029000 . hal-01275287

\section{HAL Id: hal-01275287 \\ https://hal.science/hal-01275287}

Submitted on 19 Feb 2016

HAL is a multi-disciplinary open access archive for the deposit and dissemination of scientific research documents, whether they are published or not. The documents may come from teaching and research institutions in France or abroad, or from public or private research centers.
L'archive ouverte pluridisciplinaire HAL, est destinée au dépôt et à la diffusion de documents scientifiques de niveau recherche, publiés ou non, émanant des établissements d'enseignement et de recherche français ou étrangers, des laboratoires publics ou privés. 


\title{
Inverse Patch Transfer Functions method as a tool for source field identification
}

\author{
Dorian Vigoureux \\ Nicolas Totaro* \\ Email: nicolas.totaro@insa-lyon.fr \\ Jonathan Lagneaux \\ Jean-Louis Guyader \\ Laboratoire Vibrations Acoustique \\ INSA-Lyon \\ 25 bis, av. Jean Capelle \\ F-69621 Villeurbanne Cedex \\ France
}

\begin{abstract}
Many methods to detect, quantify or reconstruct acoustic sources exist in the literature and are widely used in industry (Near-field acoustic holography, inverse Boundary Element Method, etc.). However, the source identification in a reverberant or non-anechoic environment on an irregularly shaped structure is still an open issue.

In this context, the inverse Patch Transfer Functions Method firstly introduced by Aucejo et al. [1] can be a suitable method. Indeed, the iPTF method has been developed to identify source velocity on complex geometries and in a non-anechoic environment. However, to obtain good results, the application of the method must follow rigorous criteria that were not fully investigated yet. In addition, as it was firstly defined, the iPTF method only provides source velocity while wall pressure or intensity should also give useful information to engineers.

In the present article, a procedure to identify wall pressure and intensity of the source without any additional measurement is proposed. This procedure only needs simple numerical post-processing. Using this new intensity identification, the influence of background noise, evanescent waves and mesh discretization are illustrated on numerical examples. Finally an experiment on a vibrating plate is shown to illustrate the iPTF procedure.
\end{abstract}

*Address all correspondence to this author. 


\section{Introduction}

Many methods, based on acoustic measurements, have been developed to detect and quantify noise sources. Those methods are particularly used when a direct measure on an object is not possible (because of the complexity of its geometry for example) or if one wishes to predict the noise produced by the object. Among all these methods allowing to solve the inverse problem in acoustics, the Near-field Acoustical Holography has to be cited as an example, as well as the inverse Boundary Element Method. The Near-field Acoustical Holography (NAH) was developed by J. D. Maynard et al. [2]. It permits to identify the velocity field of the source, using the pressure field measured on a hologram located near the source. Initially, this method could only be applied for the reconstruction of a planar structure and was brought to various academic shapes by E. G. Williams et al. [3,4]. One of its limitations is that it has to be applied in the near-field to prevent effects due to vanishing waves or finite 2D Fourier transform.

The inverse Boundary Elements Method (iBEM) is based on the numerical computation and inversion of transfer matrices. This method is suitable for irregularly shaped sources but suffers from a high computational cost, especially when the complexity of the structure and of the acoustical domain increases.

A hybrid method [5] has been set up using elements from both the NAH and the iBEM in order to tackle their limitations. This hNAH method is based on the least square solution of the Helmholtz equation to express the radiated sound pressure as a combination of inbound and outbound spherical waves.

The NAH can also be coupled to force distribution identification technique as presented by Pézerat et al. [6]. The objective is here to use the velocity field identified by NAH method to deduce the force distribution that produces vibration and sound radiation. The source of vibration is then quantified and localized by acoustic measurements. Another method has been recently introduced by Langrenne et al. [7,8]. This method, known as Deconfined Acoustic Holography (DAH) is based on the decomposition of the acoustic field measured on a surface surrounding the source. It states that the measured source is the composition of the free field pressure radiated by the primary source, the incoming field radiated by all secondary sources and reflected by the enclosure and the field scattered by the surface of the tested source. The separation of the outgoing field from the incoming field is performed using a Helmholtz standard integral formulation and the removing of the scattering of the incoming field is possible as the primary source is considered to be a rigid body.

We present in this paper the inverse Patch Transfer Functions method (iPTF), introduced to identify the vibration velocity on irregularly shaped sources [1] in a non-controlled acoustic environment (reverberant room, stationary disturbing sources, etc.) even in the presence of rigid obstacles (non-homogeneous acoustic medium). This inverse formulation is based on the direct formulation firstly developed by Ouisse et al. [9].

In its direct formulation (from the source to the radiated sound pressure), the PTF consists in calculating acoustic impedances of uncoupled sub-domains (cavities, structures or semi-infinite media) to predict the sound pressure or the structural velocity when all the sub-domains are coupled. The whole domain can contain sources, absorbing materials, rigid surfaces, openings, etc. To apply the PTF method, one thus proceeds to the decomposition of the global domain into sub-domains to reduce the computation time. These acoustical sub-domains are then coupled by their common surfaces divided into elementary surfaces called patches. The acoustic impedances are evaluated numerically on these patches using 
the suitable method (analytic solution, finite element method, Rayleigh's equation, measurements, etc.). Using the coupling conditions on the coupling surfaces, one obtains a system of linear equations where unknowns are the coupling velocities. Solving this system of equations leads to the possibility of computing the response at any point of the whole domain. This method, developped in the framework of the VisPeR and SILENCE european projects, has been successfully applied on industrial applications $[10,11]$ and can be a useful tool to detect efficient positions of absorbing materials to reduce noise in the domain [12].

In the direct formulation, the coupling velocity is thus deduced from a system of equations when the source is known (monopoles, velocity field for example). In the inverse formulation presented here, the source is unknown and the coupling velocity is measured on an arbitrarily defined surface surrounding the source. Using this method makes possible to identify the velocity field of the source even if the considered surface is unreachable thanks to the finite element solver used here [13].

In this article, the direct formulation will be associated to the inverse formulation to give access to information about boundary pressure and intensity on the source surface. Then, the limitations of the method will be investigated. A particular attention will be paid to the distance to the object as the radiated field partly consists of evanescent waves that cannot be measured beyond the near-field. A notion of penetration depth to estimate the bandwidth limitation of the iPTF method will be introduced.

In addition, the influence of the discretization of the identification and the measurement surfaces will be investigated and rigorous criteria will be defined. The sensitivity of iPTF to noise measurement will also be evaluated.

Finally, an experiment on the vibration of a flat plate excited by a shaker will be shown to illustrate the iPTF procedure.

\section{Theoretical background}

The method used hereby combines the direct and inverse formulation of the PTF method. We will then remind the equations used in both cases, starting with the direct formulation.

\subsection{Solving the direct problem}

As a general matter, the problem here is to compute the pressure and velocity fields at any point in a cavity due to one or more sources. Such a cavity is illustrated in Fig. 1.

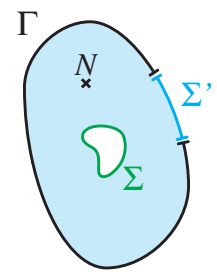

Fig. 1. Example of system under study. A vibrating surface $\Sigma$ radiates in an acoustic volume with a rigid surface $\Lambda$ and an opening $\Sigma^{\prime}$.

The source is represented by the surface $\Sigma$ vibrating with a known velocity field. In the example of Fig. $1, \Gamma$ is a rigid 
surface and $\Sigma^{\prime}$ is an opening towards an arbitrary acoustic environment. In this cavity, the acoustical problem can be solved using the Helmholtz equation

$$
\Delta p(N)+k^{2} p(N)=0 \quad \forall N \in A,
$$

where $p(N)$ is the acoustic pressure at point $N$ and $k=\omega / c$ is the wavenumber with $\omega$ the angular frequency and $c$ the sound speed in the acoustic medium. The boundary conditions are defined in Eqs. (2) and (3)

$$
\frac{\partial p(Q)}{\partial n}=0 \quad \forall Q \in \Gamma
$$

and

$$
\frac{\partial p(Q)}{\partial n}=-i \omega \rho_{0} V(Q) \quad \forall Q \in \Sigma, \Sigma^{\prime}
$$

where $\rho_{0}$ is the density of the acoustic medium and $V(Q)$ is the normal velocity on surfaces $\Sigma$ and $\Sigma^{\prime}$. To solve Eq. (1) one can use Green's theorem:

$$
\begin{aligned}
\iiint_{A}\left[\phi_{n}(N) \Delta p(N)-p(N) \Delta \phi_{n}(N)\right] d V & =\iint_{\Sigma \cup \Gamma}\left[\phi_{n}(N) \frac{\partial p(N)}{\partial n}-p(N) \frac{\partial \phi_{n}(N)}{\partial n}\right] d S,
\end{aligned}
$$

where $\phi_{n}(N)$ could be an arbitrary function (defined and twice differentiable on the domain). To simplify Eq. (4), it has been chosen to use the eigen-modes of the cavity with all boundary surfaces $\left(\Gamma, \Sigma\right.$ and $\left.\Sigma^{\prime}\right)$ considered to be rigid. This decomposition is only a mathematical tool used to solve the problem even if this is not in accordance with the physical boundary conditions. Such a decomposition is valid in PTF approach as it is a substructuring approach where sub-domains have to be solved independently from each other. In that case, for example, the opening is not represented by the modal basis of the cavity but by coupling conditions between sub-domains. An illustration of that method on two coupled cavities is shown in [9].

As $\phi_{n}(N)$ are eigen-modes of the cavity with rigid walls, they respect Helmholtz equation

$$
\Delta \phi_{n}(N)+k_{n}^{2} \phi_{n}(N)=0 \quad \forall N \in A,
$$


and boundary conditions

$$
\frac{\partial \phi_{n}(Q)}{\partial n}=0 \quad \forall Q \in \Sigma^{\prime} \cup \Sigma \cup \Gamma .
$$

Writing $p(N)$ as a sum of contributions of eigen-modes and considering the boundary conditions (Eq. (2) and (3)), one can express the pressure at any point in the cavity:

$$
p(N)=i \omega \rho_{0} \sum_{p=1}^{\infty}\left[\frac { \phi _ { p } ( N ) } { \Lambda _ { p } [ k ^ { * 2 } - k _ { p } ^ { 2 } ] } \left[\iint_{\Sigma} \phi_{p}(Q) V(Q) d Q\right.\right.
$$

$$
\left.\left.+\iint_{\Sigma^{\prime}} \phi_{p}\left(Q^{\prime}\right) V\left(Q^{\prime}\right) d Q^{\prime}\right]\right]
$$

where $\Lambda_{p}$ is the norm of the $p$-th mode, $k^{*}=k(1+i \eta), k$ is the acoustic wavenumber and $\eta$ is the damping loss factor of the fluid. As can be seen in Eq. (7), the surface $\Gamma$, which is physically rigid, does not appear in the calculation of the pressure at point $N$. This is due to the rigid wall boundary conditions we used to express the pressure that actually corresponds to real boundary conditions.

To compute Eq. (7), it is necessary to express it in a discrete form

$$
p(N)=i \omega \rho_{0} \sum_{p=1}^{\infty}\left[\frac { \phi _ { p } ( N ) } { \Lambda _ { p } [ k ^ { 2 } - k _ { p } ^ { 2 } ] } \left[\sum_{\Sigma_{e}=1}^{N_{\Sigma_{e}}}\left\langle\phi_{p}\right\rangle_{\Sigma_{e}}\langle V\rangle_{\Sigma_{e}} \mathcal{A}_{\Sigma_{e}}\right.\right.
$$

$$
\left.\left.+\sum_{\Sigma_{e}^{\prime}=1}^{N_{\Sigma_{e}^{\prime}}}\left\langle\phi_{p}\right\rangle_{\Sigma_{e}^{\prime}}\langle V\rangle_{\Sigma_{e}^{\prime}} \mathcal{A}_{\Sigma_{e}^{\prime}}\right]\right]
$$

where $\langle\chi\rangle_{S}$ is the mean of the variable $\chi$ on surface $S$, and $\mathcal{A}_{S}$ is the area of surface $S$. Eq. (8) can be reorganized as a function of velocity information. Indeed, if one isolates the terms depending on the velocity from the rest of the equation, one can write

$$
p(N)=\sum_{\Sigma_{e}=1}^{N_{\Sigma_{e}}}\langle V\rangle_{\Sigma_{e}} \cdot Z_{\Sigma_{e} \rightarrow N}+\sum_{\Sigma_{e}^{\prime}=1}^{N_{\Sigma_{e}^{\prime}}}\langle V\rangle_{\Sigma_{e}^{\prime}} \cdot Z_{\Sigma_{e}^{\prime} \rightarrow N}
$$

where

$$
Z_{\Sigma_{e} \rightarrow N}=i \omega \rho_{0} \sum_{p=1}^{\infty} \frac{\mathcal{A}_{\Sigma_{e}} \phi_{p}(N)\left\langle\phi_{p}\right\rangle_{\Sigma_{e}}}{\Lambda_{p}\left[k^{* 2}-k_{p}^{2}\right] .}
$$


The term $Z_{\Sigma_{e} \rightarrow N}$ is called acoustic impedance. It permits to calculate the pressure at a point $N$ using the velocities of a series of surfaces $\Sigma_{e}$ called patches. The notation $\Sigma_{e} \rightarrow N$ indicates that we consider the transmission path from the patch $\Sigma_{e}$ to the point $N$. Eq. (9) shows that the radiated pressure can be computed using the velocity of the vibrating surface $\Sigma$ and the velocity of the opening surface $\Sigma^{\prime}$. To solve the direct problem, the velocity on $\Sigma$ is supposed to be known and the velocity on $\Sigma^{\prime}$ is deduced from coupling conditions between sub-domains as presented in [9]. The basic idea of the inverse Patch Transfer Function is included in Eq. (9). Indeed, if the coupling velocity $\langle V\rangle_{\Sigma_{e}^{\prime}}$ is not unknown but measured all the informations of the outer sub-domain are taken into account and the pressure $p(N)$ can be computed at any point of the sub-domain. If the effective velocity on coupling surface $\langle V\rangle_{\Sigma_{e}^{\prime}}$ is known, the modelization of acoustic environment outside the acoustic volume is not necessary but intrinsically taken into account by measurements.

\subsection{Solving the inverse problem}

Eq. (9) permits to compute the pressure at any point of the sub-domain and particularly on the coupling surface $\Sigma^{\prime}$. Averaging this pressure on an elementary surface $\Sigma_{e}^{\prime}$, one can express the following system of equation

$$
\left\{\langle P\rangle_{\Sigma_{e}^{\prime}}\right\}=\left[Z_{\Sigma_{e} \rightarrow \Sigma_{e}^{\prime}}\right]\left\{\langle V\rangle_{\Sigma_{e}}\right\}+\left[Z_{\Sigma_{e}^{\prime} \rightarrow \Sigma_{e}^{\prime}}\right]\left\{\langle V\rangle_{\Sigma_{e}^{\prime}}\right\}
$$

where the patch to patch impedance is defined as

$$
Z_{\Sigma_{e} \rightarrow \Sigma_{e}^{\prime}}=i \omega \rho_{0} \sum_{p=1}^{\infty} \frac{\mathcal{A}_{\Sigma_{e}}\left\langle\phi_{p}\right\rangle_{\Sigma_{e}}\left\langle\phi_{p}\right\rangle_{\Sigma_{e}^{\prime}}}{\Lambda_{p}\left[k^{* 2}-k_{p}^{2}\right]}
$$

The general purpose of the inverse problem is to compute the velocity field of the source based on measurements of the radiated field. Starting from Eq. (11), the velocities of elementary surfaces $\Sigma_{e}$ of the source can be expressed as a function of acoustic patch impedance matrices and patch pressure and patch acoustic velocity on elementary surfaces $\Sigma_{e}^{\prime}$ of the opening:

$$
\left\{\langle V\rangle_{\Sigma_{e}}\right\}=\left[Z_{\Sigma_{e}^{\prime} \rightarrow \Sigma_{e}}\right]^{-1}\left(\left\{\langle P\rangle_{\Sigma_{e}^{\prime}}\right\}-\left[Z_{\Sigma_{e}^{\prime} \rightarrow \Sigma_{e}^{\prime}}\right]\left\{\langle V\rangle_{\Sigma_{e}^{\prime}}\right\}\right)
$$

The acoustic patch impedance matrices of the volume defined by surfaces $\Sigma$ and $\Sigma^{\prime}$ are computed using Eq. (12) and eigen-modes extracted with standard finite element solver. The pressure and velocity vectors of Eq. (13) are measured on the virtual surface $\Sigma^{\prime}$ using $\mathrm{pU}$ probe for example.

The geometry of the acoustic volume is defined by the shape of the object under study $(\Sigma)$, by the presence of rigid walls $(\Gamma)$ and by the measurement surface $\left(\Sigma^{\prime}\right)$. If no rigid wall physically exists, the measurement surface $\Sigma^{\prime}$ has to completely surround the source. In that case, $\Sigma^{\prime}$ can be chosen arbitrarily and the acoustic volume is virtual (not defined by a physical 
surface). In the following, it is assumed that the exact geometry of the vibrating object is known and that the surface $\Sigma$ corresponds to it. However, there is no theoretical need for this and the surface $\Sigma$ can be defined arbitrarily as, for example, a sphere or an ellipsoid around the source. Surface $\Sigma$ is a surface of identification of particle velocity in the acoustic medium. If the surface of identification corresponds to the surface of the source, one considers, thanks to continuity conditions, that the particle velocity is equal to the source velocity.

Eq. 13 has the classical form of an ill-posed problem. The solution is found using Tikhonov regularization and the maximum of curvature of the L-curve $[14,15]$.

Using this identified velocity field, the identification of pressure and intensity fields on the source surface is also possible. This identification can be performed by combining both direct and inverse formulations of the PTF method.

Starting from the pressure and velocity fields measured on the opening surface $\Sigma^{\prime}$, the velocity field of the source can be computed using Eq. (13). At this step, the velocity field of the source is identified as already presented in [1].

To go further we combine the identified velocity field to Eq. (9). Indeed, as Eq. (9) is true for any point N, one can now compute the pressure on the source surface, using the velocity $\langle V\rangle_{\Sigma_{e}^{\prime}}$ measured and the velocity $\langle V\rangle_{\Sigma_{e}}$ computed during step 1. The wall pressure of the source can then be written

$$
\left\{\langle P\rangle_{\Sigma_{e}}\right\}=\left[Z_{\Sigma_{e} \rightarrow \Sigma_{e}}\right]\left\{\langle V\rangle_{\Sigma_{e}}\right\}+\left[Z_{\Sigma_{e}^{\prime} \rightarrow \Sigma_{e}}\right]\left\{\langle V\rangle_{\Sigma_{e}^{\prime}}\right\}
$$

Finally, the intensity field is easily obtained using the usual equation

$$
\left\{\langle I\rangle_{\Sigma_{e}}\right\}=\frac{1}{2} \operatorname{Re}\left[\left\{\langle P\rangle_{\Sigma_{e}}\right\}\left\{\langle V\rangle_{\Sigma_{e}}\right\}^{*}\right]
$$

where $\left\{\langle P\rangle_{\Sigma_{e}}\right\}$ and $\left\{\langle V\rangle_{\Sigma_{e}}\right\}$ are the pressure and velocity fields computed during steps 1 and 2 and $*$ represents the transpose complex conjugate.

With this new step, the source is completely characterized (velocity, wall pressure, intensity, power, radiation efficiency). Obviously, the acoustic particle velocity is the primary unknown and its bad estimation will lead to wrong estimations of the other quantities.

\section{Numerical validation of the intensity identification}

For this numerical validation, we present the identification performed on a baffled steel plate excited by a harmonic point force as presented in Fig. 2. The plate is $0.45 \mathrm{~m}$ long, $0.35 \mathrm{~m}$ large and $5 \mathrm{~mm}$ thick and it is made of steel (Young's modulus $E=2.1 e 11 \mathrm{~Pa}$; density $\rho=7800 \mathrm{~kg} . \mathrm{m}^{3}$; Poisson's ratio $\mathrm{v}=0.3$; damping $\eta=0.02$ ). The plate is excited by an unit point force located at point $(0.1 ; 0.1) \mathrm{m}$ on the frequency band $[5: 4000] \mathrm{Hz}$ (frequency step $5 \mathrm{~Hz}$ ). All modes up to $8000 \mathrm{~Hz}$ 


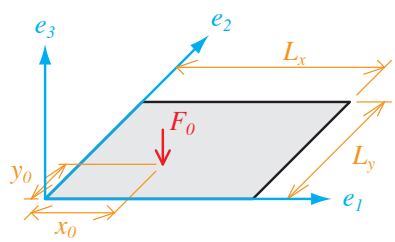

Fig. 2. System under study: a plate excited by a point force radiating into a semi-infinite acoustic medium.

are used to computed the vibro-acoustic response of the plate (427 modes).

\subsection{Measurement step (numerical experiment)}

The measurement step consists in the measurement of both pressure and velocity fields on the opening surface $\Sigma^{\prime}$. For this first application, the opening surface is defined as shown on Fig. 3. We are here in a particular case where the rigid wall $\Gamma$ is reduced to null and the opening is entirely surrounding the source. It is important to notice here that the radiated field

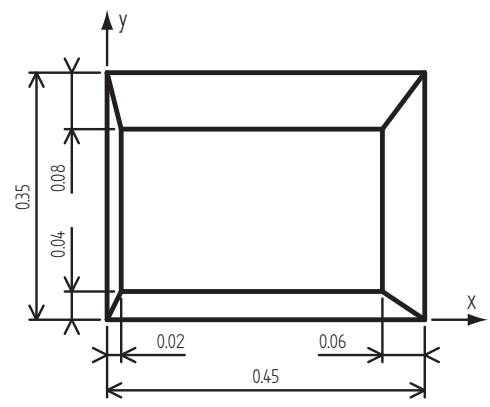

Fig. 3. Definition of the virtual surface $\Sigma^{\prime}$ surrounding the rectangular plate. Top view. Heigth $=0.045 \mathrm{~m}$.

obtained at the measurement step has been provided by a numerical experiment performed using a boundary element method in infinite domain avoiding noise associated to real experiments and permitting to clearly state validity domain of the method and define rigorous criteria needed.

\subsection{Identification step}

We focus here on the normal intensity identified on the source surface. Fig. 4 shows two different maps of the intensity field. The first one (Fig. 4(a)) represents the reference field computed using Boundary Element Method while the second one (Fig. 4(b)) represents the intensity identified on the plate using the iPTF algorithm based on the radiated pressure and velocity fields computed during the numerical experiment. These maps show a good agreement between the reference and the identified intensity field. Besides, amplitudes and positions of maximum are well estimated. These two maps are given at a particular frequency. It is then important to look out for the intensity identification in the whole frequency range. The identified averaged intensity $\bar{I}_{\text {identified }}(\omega)$ is compared to the reference averaged intensity $\bar{I}_{\text {ref }}(\omega)$ as a function of frequency in Fig. 5(a). This figure shows that the intensity is properly identified although there are some discrepancies existing between $100 \mathrm{~Hz}$ and $1800 \mathrm{~Hz}$. This can be seen more precisely on Fig. 5(b) that represents the identification error as defined in Eq. 


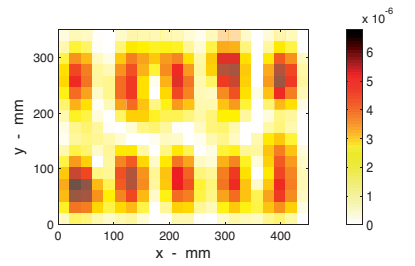

(a)

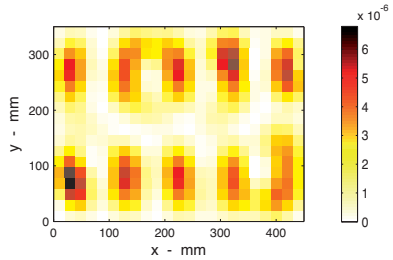

(b)

Fig. 4. Intensity fields at $1900 \mathrm{~Hz}$. (a) Reference field; (b) Identified field.

(16):

$$
\varepsilon(\omega)=10 \times \log _{10}\left(\bar{I}_{\text {ref }}(\omega)\right)-10 \times \log _{10}\left(\bar{I}_{\text {identified }}(\omega)\right) .
$$

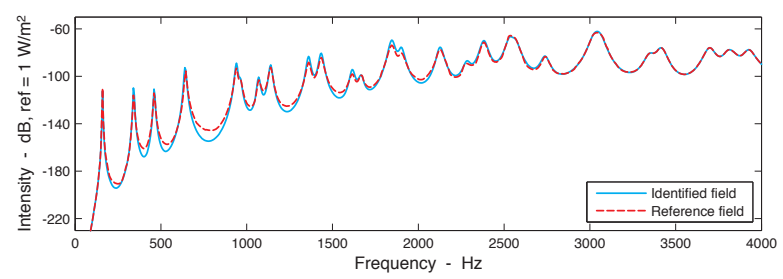

(a)

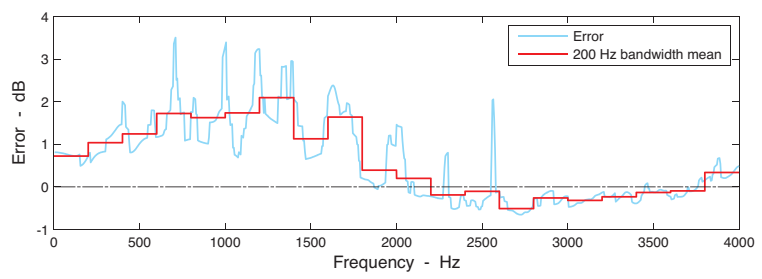

(b)

Fig. 5. Space averaged intensity on the surface of the plate as a function of frequency. (a) Comparison of reference and identified intensity; (b) Identification error in $\mathrm{dB}$.

One can see on this figure that the error is less than $1 \mathrm{~dB}$ for frequencies higher than $1800 \mathrm{~Hz}$. Errors in low frequencies are usually due to evanescent waves in the radiated field. We will then study their influence in the identification of the intensity field using the iPTF method.

\section{Effect of the evanescent waves}

It is widely known that every structure presents an evanescent radiation below the critical frequency. In this article, we want to study how those evanescent waves are influencing the application of the iPTF method. For this purpose, we will remind here some fundamental equation of plate vibration in order to define a parameter called penetration depth. 
The pressure radiated by an infinite plate in a semi-infinite medium is given by [16]

$$
p(x, y, z)=-i \frac{\omega^{2} \rho_{0} w(x, y)}{k_{z}} \cdot e^{i k_{z} z}
$$

The penetration depth $d$ corresponds here to the distance $z$ at which the radiated pressure amplitude is divided by $e=2.718$ compared to the boundary pressure. It is the distance at which the exponential is equal to $e^{-1}$ and is expressed as

$$
d=\frac{1}{\frac{\omega}{c} \sqrt{\frac{\omega_{c}}{\omega}-1}}
$$

Fig. 6 shows the penetration depth versus frequency. One can see that very low frequency waves can propagate far from

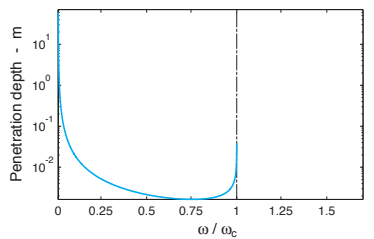

(a)

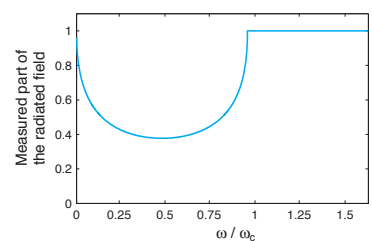

(b)

Fig. 6. Influence of the evanescent waves. (a) Penetration depth of evanescent waves of an infinite plate as a function of $\omega / \omega_{c}$; (b) reduction factor at the distance $z_{s}$ from the plate as a function of $\omega / \omega_{c}$.

the plate thanks to their large wavelengths. Then, the penetration depth quickly decreases as a function of wavelength. Thus, the ability to measure evanescent waves decreases while the frequency increases, in such a way that for $\omega / \omega_{c}>0.18$, being farther than $10 \mathrm{~cm}$ makes it difficult to measure the evanescent waves especially if the signal to noise ratio is high. When reaching the critical frequency, the penetration depth slightly increases. Above critical frequency, waves are propagative and the penetration depth is undefined.

Conversely, at a given distance $z_{s}$ from the plate, the reduction factor $e^{-z_{s} / d}$ indicates the part of the initial amplitude (at $z=0$ ) that is measured at the distance $z_{s}$ from the plate. This factor is visualized in Fig. 6(b). In this figure, one can identify two different behaviors, below and above critical frequency. Above $f_{c}$, the reduction factor is close to one that indicates that the amplitude measured at $z_{s}$ is approximately equal to the one at $z=0$. Below $f_{c}$ and because of the decrease of evanescent waves, their amplitudes are highly reduced. Obviously, this will have a consequence on the quality of iPTF results mainly because of the measurement noise and the regularization process. In particular, the amplitude of the real velocity of the source might be underestimated in this frequency band.

The agreement of the penetration depth criterion with results presented in Fig. 5(b) is very good. One can see that the more vanishing is the field, the greater is the identification error. As any method based on acoustical measurement, it is 
proven here that the iPTF method is limited by the knowledge of the evanescent part of the radiated field. Above the critical frequency, the radiated field is essentially propagative, allowing thus an identification with a low error (about $0.5 \mathrm{~dB}$ ).

These remarks, based on an infinite plate analogy, are guidelines. In the case of a finite plate, each mode has its own radiation efficiency.

Fig. 7(a) and Fig. 7(b) show differences between the reference field and the identified field. As can be seen, both maps are slightly different, the identified one seems to be blurred and less details are visible. As explained previously, this is probably due to the evanescent part of the radiated field. To prove this conjecture, it is interesting to compute the field that could be radiated by these two different fields (reference and identification). Fig. 7 presents the following information: (i) the reference boundary velocity field, Fig. 7(a); (ii) the identified boundary velocity field, Fig. 7(b); (iii) the velocity field radiated at 4 and $20 \mathrm{~cm}$ by the reference field, Fig. 7(c); (iv) the velocity field radiated at 4 and $20 \mathrm{~cm}$ by the identified field, Fig. 7(d). These fields are displayed here at $710 \mathrm{~Hz}$ where, as can be seen on Fig. 5(b), the error is the highest. Indeed, Fig.

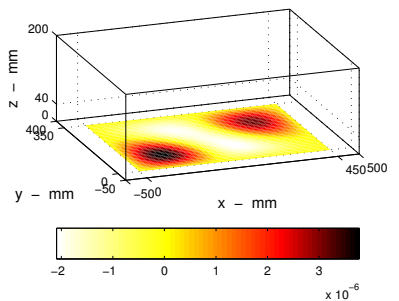

(a)

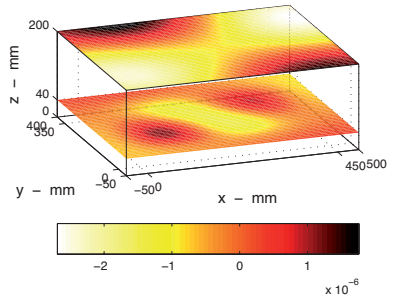

(c)

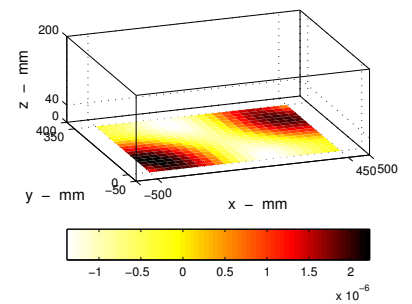

(b)

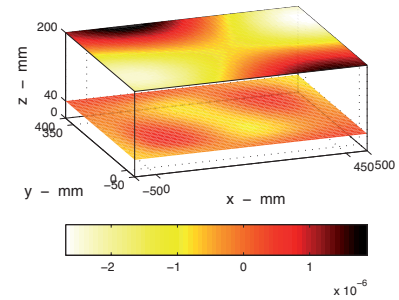

(d)

Fig. 7. Velocity field (a) computed on the surface of the plate (reference), (b) identified by iPTF and radiated velocity fields computed using (c) reference or (d) the identified field at 4 and $20 \mathrm{~cm}$ from the plate at $710 \mathrm{~Hz}$.

7(a) and 7(b) are highly different. However, these two very different velocity fields produce nevertheless the same radiated velocity at $20 \mathrm{~cm}$, as presented in Fig. 7(c) and 7(d). Contrary, in the near-field (plane at $4 \mathrm{~cm}$ ), the noticeable discrepancies are due to the influence of evanescent waves. Using the iPTF method, the identification is performed using the radiated field measured on a surface at a certain distance from the source. Due to the existence of the evanescent waves, the identified field can only represent a part of the exact field. That is the part which leads to radiations in the far-field. Even if the identified field shown on Fig. 7(b) is not the exact field that could be measured directly on the plate, it is the part responsible for the radiations that effectively contribute to the sound power in the far-field called supersonic acoustic intensity by Williams [17] 
and Fernandez et al. [18] or useful intensity by Corrêa et al. [19].

\section{Effect of the meshes}

The iPTF method is based on a discrete decomposition of the identification and measurement surfaces. Therefore, some errors may occur regarding the discretization used. The results shown here illustrate the influence of the meshes on the identification performed with the iPTF method.

\subsection{Discretization of the identification surface with bending waves criterion}

Fig. 8 shows the different meshes used for the identification. They are respectively made using a $\lambda_{b} / 2, \lambda_{b} / 4$ and $\lambda_{b} / 6$ criterion, where $\lambda_{b}$ is the wavelength of the bending waves in the plate. The measurement mesh is made using the $\lambda_{a} / 4$ criterion, where $\lambda_{a}$ is the acoustical wavelength in air. This measurement mesh is considered here as fine enough to ensure a good convergence of results (see §5.2) and evaluate the influence of discretization of identification mesh. Fig. 9 presents

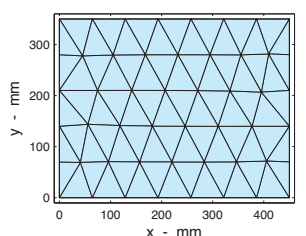

(a)

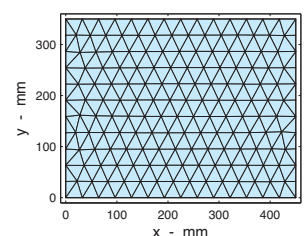

(b)

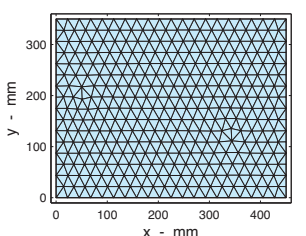

(c)

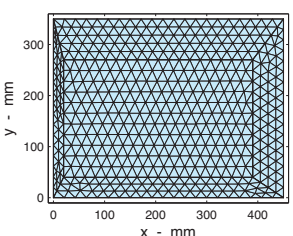

(d)

Fig. 8. Identification and measurement meshes used in the study on influence of the identification mesh. (a) Identification mesh - $\lambda_{b} / 2$; (b) Identification mesh $-\lambda_{b} / 4$; (c) Identification mesh $-\lambda_{b} / 6$; (d) Measurement mesh $-\lambda_{a} / 4$.

the results obtained with these three identification meshes. Fig. 9(a) is the reference map, computed numerically. The

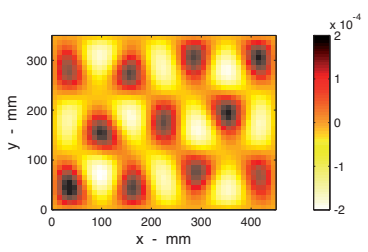

(a)

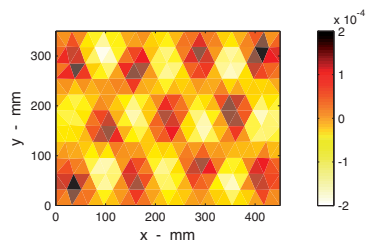

(c)

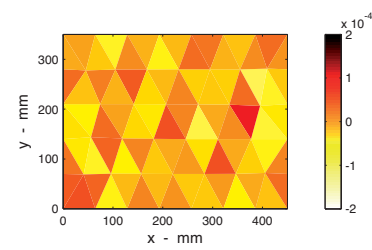

(b)

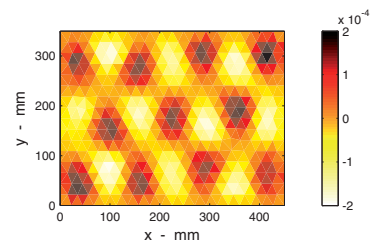

(d)

Fig. 9. Velocity fields identified at $3900 \mathrm{~Hz}$ with the different identification meshes shown in Fig. 8. (a) Reference field; (b) identified field obtained with $\lambda_{b} / 2$ mesh; (c) identified field obtained with $\lambda_{b} / 4$ mesh; (e) identified field obtained with $\lambda_{b} / 6$ mesh (963 eigen modes).

identification is performed using the iPTF algorithm based on the simulated pressure and velocity radiated fields. Fig. 9(b) 
shows clearly that the $\lambda_{b} / 2$ mesh is quite insufficient to accurately represent the physical phenomena that are taking place. In this first application, neither the localization is precise nor the amplitude is correctly evaluated, mostly due to the large areas on which the mean amplitude is calculated. The correlation between the $\lambda_{b} / 4$ identification and the reference is much better. One can easily see, on Fig. 9(c), the nodal lines of the plate. Besides, the values of the velocity are identified with a higher precision. Same as previously, the extrema are lowered by the effect of the mean but as the area of the patches is smaller, this error has a lower effect on the identification of the velocity. Regarding the increase of precision while meshing with a $\lambda_{b} / 4$ criterion instead of a $\lambda_{b} / 2$ criterion, one could think that a $\lambda_{b} / 6$ would lead to better results. This is not the case. The mesh fineness is limited to a certain point beyond which the results become incoherent. Indeed, the iPTF method consists in the inversion of a system whose size is given by the parameters of the problem. The number of unknowns is equal to the number of points where one wishes to compute the velocity, the number of equations is equal to the number of points at which the radiated pressure and velocity have been measured. Thus, the problem can be over or under determined and then treated in least square sense. To verify that the number of independent equations is effectively equal to the number of measurement points, the rank of the matrix to be inverted is computed. Tab. 1 shows the rank of the system for the three meshes. For the first two cases, one can see that the equations are linearly independent whereas the equations of the third

Table 1. Rank of the systems

\begin{tabular}{ccc}
\hline Mesh & Number of unknowns & Rank \\
\hline$\lambda_{b} / 2$ & 74 & 74 \\
$\lambda_{b} / 4$ & 316 & 316 \\
$\lambda_{b} / 6$ & 664 & 427 \\
\hline
\end{tabular}

system are dependent. The rank of this system is lower than the number of its equations and is not limited by the number of available equations. As said in $\S 2.1$, the problem is decomposed on the cavity eigen-modes basis computed up to 8000 $\mathrm{Hz}$. In this frequency band, the number of modes of the virtual cavity is 427. It appears clearly that the rank of the system is limited by the number of modes taken into account in impedances computation. As a consequence, the number of modes must be at least equal to the number of identification points. To verify this hypothesis, we increased the modal basis to modes up to $11 \mathrm{kHz}$. Doing this, 963 modes are taken into account. The application of the iPTF method on the former data with this extended modal basis leads to the result shown in Fig. (9(d)). One can see on this figure that the vibration field is now identified with a good accuracy.

\subsection{Discretization of the measurement surface with acoustic wave criterion}

Fig. 10 shows the different meshes used for the measurement. They are respectively made using a $\lambda_{a} / 2, \lambda_{a} / 4$ and $\lambda_{a} / 6$ criterion, where $\lambda_{a}$ is the acoustical wavelength in air. The identification mesh shown on Fig. 10(d) is made using the $\lambda_{b} / 4$ criterion, where $\lambda_{b}$ is the wavelength of the bending waves in the plate (seen to produce good results in §5.1). Fig. 11 presents the results obtained with these three measurement meshes. Fig. 11(a) is the same as Fig. 9(a). It is the reference map of the velocity field of the plate. Figs. 11(b), 11(c) and 11(d) are the maps identified using the meshes hereby described. One can 


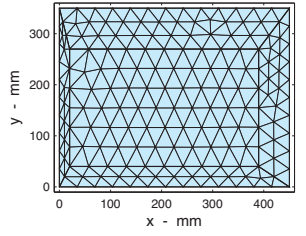

(a)

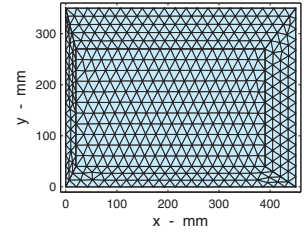

(b)

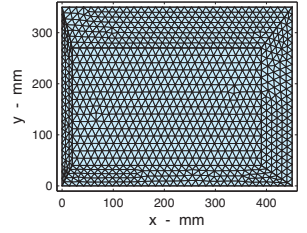

(c)

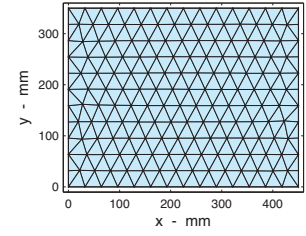

(d)

Fig. 10. Identification and measurement meshes used in the study on influence of the measurement mesh. (a) Measurement mesh $-\lambda_{a} / 2$; (b) Measurement mesh $-\lambda_{a} / 4$; (c) Measurement mesh $-\lambda_{a} / 6$; (d) Identification mesh $-\lambda_{b} / 4$.

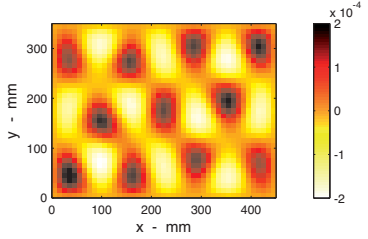

(a)

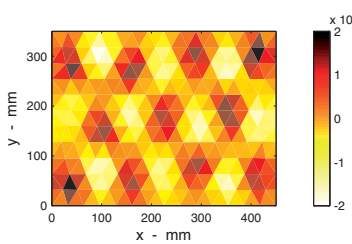

(c)

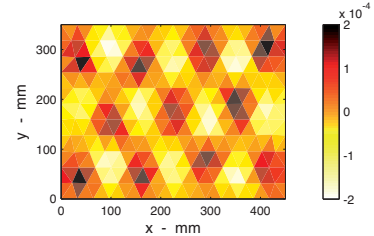

(b)

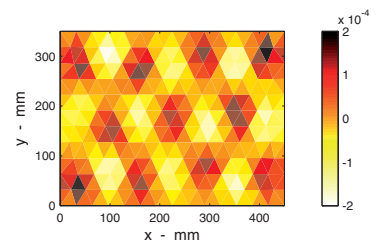

(d)

Fig. 11. Velocity fields identified at $3900 \mathrm{~Hz}$ with the different measurement meshes. (a) Reference field; (b) identified field obtained with $\lambda_{a} / 2$ mesh; (c) identified field obtained with $\lambda_{a} / 4$ mesh; (d) identified field obtained with $\lambda_{a} / 6$ mesh.

see that the three maps show equivalent results. This indicates that the measurement mesh and the number of measurement points are of little importance in the accuracy of the description of the sources and an under-determined problem can be solved here.

\subsection{Creating a mesh}

Considering the remarks presented here about the importance of the measurement and the identification step, one can define some procedure to obtain a correct identification using the iPTF method. Let call $f_{\text {max }}$ the maximal frequency of identification. In order to ensure a correct visualization of the physical phenomena, it is important to choose a patch size for the identification mesh that will be small enough to render them with a sufficient precision. Thus, the identification patches should not be larger than the fourth of the bending wavelength in the structure.

Once the size of the identification patches is chosen, and knowing the identification area, it is possible to calculate the number of identification patches $N_{\Sigma_{e}}$. The number of modes $N_{m}$ that have to be taken into account in the modal basis of the cavity is directly linked to the number of identification patches as it must necessarily be greater than $N_{\Sigma_{e}}$ to ensure the linear independence of the equations. Finally, the number of measurement patches must be equal or higher than $N_{\Sigma_{e}}$.

Summarizing: (i) The number of identification patches $N_{\Sigma_{e}}$ could be arbitrarily chosen but a $\lambda_{b} / 4$ or $\lambda_{b} / 6$ criterion is a good choice; (ii) The number of modes of the virtual cavity $N_{m}$ has to be higher than $N_{\Sigma_{e}}$; (iii) The number of measurement patches 
$N_{\Sigma_{e}^{\prime}}$ can be lower (under-determined case) or higher (over-determined case) than $N_{\Sigma_{e}}$.

\section{Influence of measurement noise}

The most common way to simulate noise during the measurement of a signal $\chi$ is to blur it using two independent gaussian variables, corresponding to the multiplicative and additive errors (respectively $\alpha$ and $\eta$ ) [20].

$$
\chi_{\text {noisy }}=\alpha \cdot \chi+\eta
$$

Most of the time, the additive part of the error is uncorrelated from the signal itself and can be suppressed using different methods such as the Principal Component Analysis [21] for example. For this reason, we will focus here on the modelization of the multiplicative error. We here develop $\alpha$ so as to let appear the percentage error $p$

$$
\begin{aligned}
\chi_{\text {noisy }} & =\chi \cdot(p+1) \cdot e^{i \Delta \varphi} \\
& =|\chi| \cdot(p+1) \cdot e^{i(\varphi+\Delta \varphi)}
\end{aligned}
$$

where $p$ and $\Delta \varphi$ are respectively the amplitude percent error and the phase error. If one consider the amplitude error $\Delta \chi=p \cdot|\chi|$ instead of the percent error, it is possible to choose those two errors at random using the normal laws.

Using this modelization is to consider that the measured value is located in a circle centered on the theoretical value and whose radius characterizes the preciseness of the measurement instrument. Both parameters $\Delta \chi$ and $\Delta \varphi$ can be used to adjust the severity of the error.

Fig. 12 shows the reference and the identified velocity power spectra when the error is applied on the measured velocity (Fig. 12(a)) or on the measured pressure (Fig. 12(b)) independently. For this simulation, an error of $3 \%$ has been used. One can see on these figures that the identification error is not particularly important even if the measurement error is important ( $\sim 2 \mathrm{~dB}$ ). The most important thing to notice is that beyond $3000 \mathrm{~Hz}$, the identified velocity is still correct when the error is made on the measured velocity whereas it is not when the error is made on the measured pressure. This let see that it is more important to ensure a good pressure measurement. This phenomenon can ben explained from Eq. (13). As

shown in this equation, there are two source terms: $\left\{\langle P\rangle_{\Sigma_{e}^{\prime}}^{A}\right\}$ and $\left[Z_{\Sigma_{e}^{\prime} \leftarrow \Sigma_{e}^{\prime}}^{A}\right]\left\{\langle V\rangle_{\Sigma_{e}^{\prime}}^{A}\right\}$. The pressure term comes directly from the measurements and is blurred by the measurement error while the velocity term is balanced by the impedance matrix computed numerically. This balance smoothes the velocity term, leading to better results in the identification when the error is committed on the velocity. 


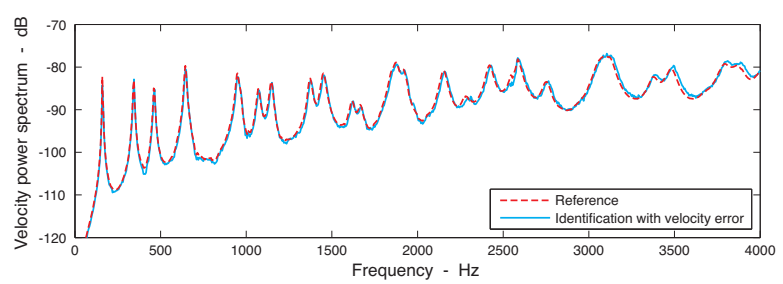

(a)

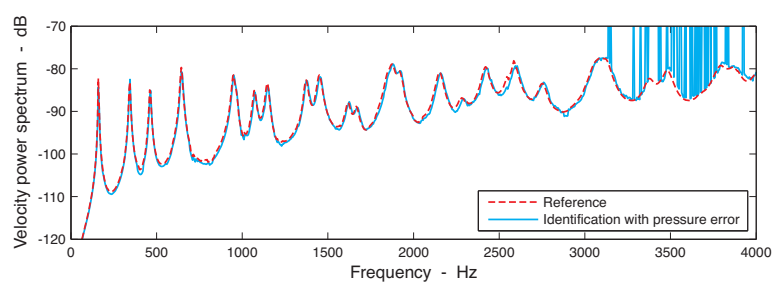

(b)

Fig. 12. Identification of the velocity when gaussian noise is added on (a) measured velocity or (b) measured pressure.

\section{Experimental validation}

An experimental validation has been performed to illustrate the previous numerical simulation. As can be seen in Fig. 13(a), a rectangular plate made of aluminum has been glued to a wood frame on $4 \mathrm{~cm}$ on the edges of the plate. This 2 $\mathrm{mm}$ thick plate is $0.68 \mathrm{~m}$ long, $0.38 \mathrm{~m}$ wide and has thus a free surface of dimensions $0.6 \times 0.3 \mathrm{~m}$. The plate is excited by an electro-dynamic shaker fed with a withe noise and radiates into a quiet room. It is important to underline here that this room is not an anechoic chamber and that no particular treatement has been used to prevent reflections on facilities (on the cartesian robot used to move sensors for example). These measurements are then not in free field conditions.

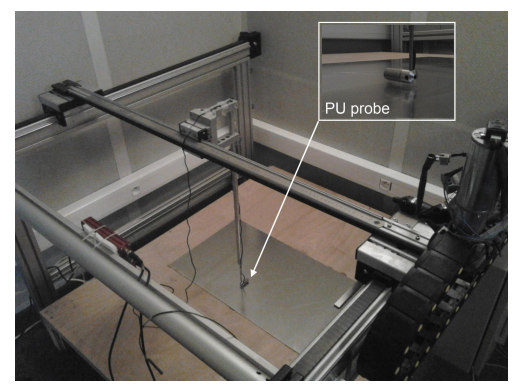

(a)

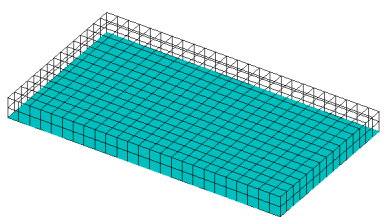

(b)

Fig. 13. System under study: an aluminum plate glued to a wood frame and excited by a shaker. (a) Photography of the system; (b) Measurement mesh surrounding the plate.

The vibration and boundary pressure fields on the plate surface have been measured directly with a laser vibrometer and a microphone (at $1 \mathrm{~mm}$ from the plate) on a regular mesh of 10 by 17 points on the whole surface of the plate. These direct measurements will be considered as the reference fields to evaluate the iPTF method. As the measurements are not synchronous, a phase reference is necessary. For sake of simplicity, the force sensor on the electro-dynamic shaker has been used as a reference. Obviously, any kind of sensor can be used in the same way (accelerometers, microphones). The reference measurements of the boundary pressure and particle velocity are represented in Fig. 14 by the solid black lines. 


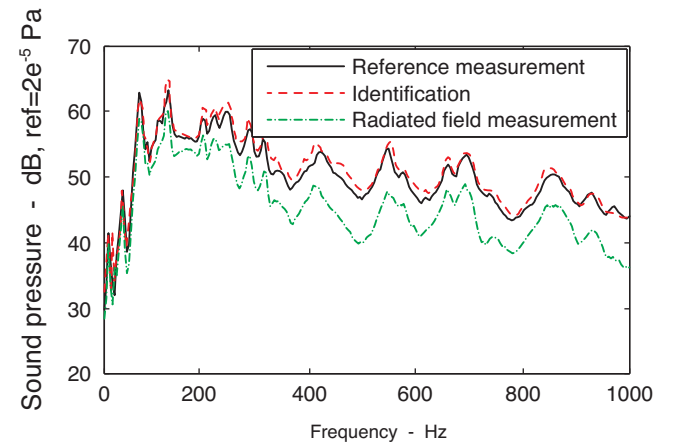

(a)

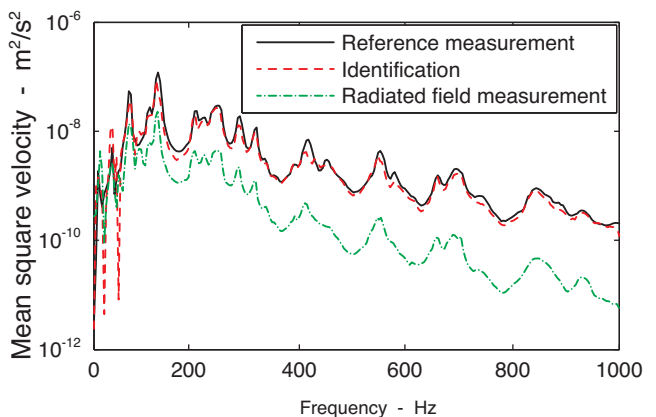

(b)

Fig. 14. Experimental comparison between reference, identified and radiated fields as function of frequency. (a) Sound pressure; (b) mean square velocity

To apply the iPTF method, an acoustic measurement surface surrounding the plate has been defined and discretized into 535 measurements points as presented in Fig. 13(b). The top surface of the acoustic measurement surface is at $5 \mathrm{~cm}$ from the plate. As can be seen in Fig. 13(a), the sound pressure and the particle velocity have been measured using a PU probe at the center of each patch constituting the acoustic measurement surface. It is considered here that the value of acoustic quantities at the center of the patch is representative of the mean value on the patch. The measured velocity is always normal to the considered surface and the same phase reference (the force sensor) has been used for acoustic measurements. The mean quadratic pressure and velocity measured on the acoustic measurement surface are also plot in Fig. 14 (dash-dotted green lines) to show that, at this distance of measurements, the differences between boundary and radiated fields are important.

Using measured pressure and particle velocity in the frequency domain and acoustic impedance computed with the FE model of the virtual volume, the pressure, velocity and intensity fields on the surface of the plate have been identified with Eq. (12).

For the sake of comparison, it has been chosen that the identification mesh will be the same as the reference mesh (170 patches). In addition, considering the number of measurements patches (535), the modal basis of the virtual cavity must have more than 535 modes. In the following, the modal basis of the virtual cavity contains all modes up to $11 \mathrm{kHz}$ (2102 modes) to avoid any problem of convergence.

The identification results are plotted in Fig. 14 (mean quadratic quantities) and Fig. 15 (maps at one particular frequency). Analysing these figures, it can be concluded that the method correctly predict mean quadratic velocity, mean quadratic pressure and radiated power in the whole frequency range under investigation. The particle velocity, which is the primary unknown, is very well estimated and the deduced unknowns (pressure and radiated power) show a good agreement with reference measurements. In Fig. 15 the comparison of the pressure, normal velocity and intensity fields directly measured on the plate with the laser vibrometer and the microphone at a particular frequency are presented. The identified maps shown in Fig. 15 give also a good representation of the source field in level as well as in space distribution. Indeed, the two intensity spots of the source are well estimated and localized. To quantify objectively the quality of the identification, the 


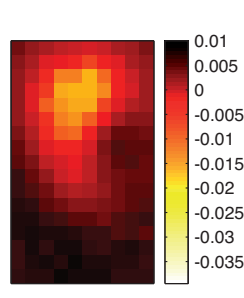

(a)

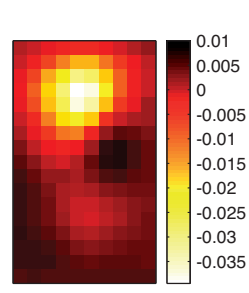

(d)

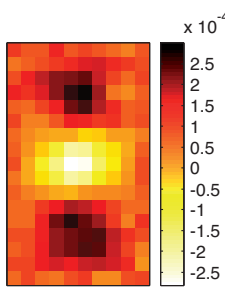

(b)

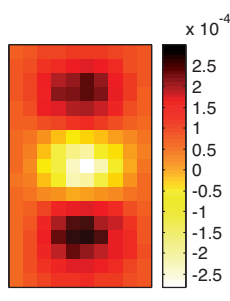

(e)

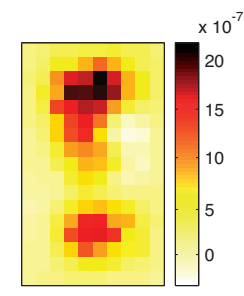

(c)

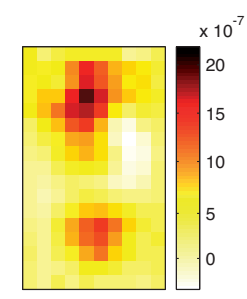

(f)

Fig. 15. Experimental comparison between reference and identified fields at $205 \mathrm{~Hz}$ on the plate surface. (a) real part of the reference pressure field; (b) real part of the reference velocity field; (c) reference active intensity field; (d) real part of the idenfied pressure field; (e) real part of the identified velocity field; (f) identified active intensity field.

Frequency Domain Assurance Criterion (FDAC, Eq. (21)) can be computed.

$$
F D A C(\omega)=\frac{\left\{V_{\text {ref }}(\omega)\right\}^{T}\left\{V_{i d}(\omega)\right\}}{\sqrt{\left\{V_{\text {ref }}(\omega)\right\}^{T}\left\{V_{\text {ref }}(\omega)\right\}\left\{V_{i d}(\omega)\right\}^{T}\left\{V_{i d}(\omega)\right\}}}
$$

where $\left\{V_{\text {ref }}(\omega)\right\}$ and $\left\{V_{i d}(\omega)\right\}$ are vectors of reference and identified velocities at angular frequency $\omega$. FDAC values are included in [-1 1]. A value of 0 indicates no correlation between reference and identified maps, a value of 1 indicates a perfect correlation and a value of -1 a perfect correlation with a phase shift of $\pi$. In the case of Fig. 15, the FDAC value is 0.9336 that indicates a really good correlation between velocity maps.

On the whole frequency band between 20 and $1000 \mathrm{~Hz}$, FDAC is most of the time higher than 0.8 as can be seen in Fig. 16. Usually correlation between maps are considered to be good for FDAC values higher than 0.75 . However, below $100 \mathrm{~Hz}$ some phase inversions can be noticed (negative values). These phase inversions might be due to a wrong calibration of the PU probe in low frequency where sensitivities of sensors vary quickly (sensitivities are frequency dependent). Above 100Hz, the identification produces really good results and validates the whole procedure of iPTF method.

\section{Conclusion}

In this article, iPTF has been used to identify both pressure and velocity fields, yielding the parietal intensity. Until now, the iPTF method has mainly been used to identify the velocity field on the surface of a structure. The mathematical formulation combining the direct and the inverse formulation in order to identify the parietal pressure and intensity fields has been presented. The first results have shown a frequency band for which the iPTF method was not giving exact results mainly due to the presenec of evanescent waves. A parameter called penetration depth has been defined. It explains why 


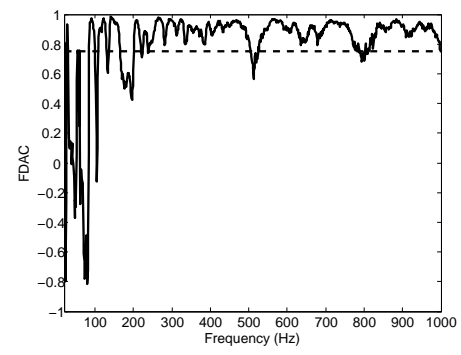

Fig. 16. Frequency Domain Assurance Criterion between reference and idenfitied velocity fields as a function of frequency. The dashed line indicates the threshold of good correlation (0.75).

identification results underestimate reality in this particular frequency band.

The influence of the discretization of the measurement and identification meshes has been studied. It has been shown that the fineness of the identification mesh is limited by the number of modes taken into account for the decomposition of the problem on the cavity eigen-modes basis.

The stability of the iPTF method regarding measurement errors has been studied. As this method is based on the measurement of both acoustical pressure and particle velocity it is interesting to know which of these two quantities was the most sensitive to noise errors. It has been demonstrated that it is more important to ensure a good pressure measurement than a good velocity measurement.

At last, the validation experiment is an example of a successful application of the iPTF method following the rigorous criteria previously defined.

\section{Acknowledgements}

This work was performed within the framework of the Labex CeLyA of Université de Lyon, operated by the French National Research Agency (ANR-10-LABX-0060/ ANR-11-IDEX-0007).

\section{References}

[1] Aucejo, M., Totaro, N., and Guyader, J.-L., 2010. "Identification of source velocities on 3d structures in non-anechoic environments: Theoretical background and experimental validation of the inverse patch transfer functions method". $J$. 
Sound Vib., 329, pp. 3691-3708.

[2] Maynard, J. D., Williams, E. G., and Lee, Y., 1985. "Nearfield acoustic holography: I. theory of the generalized holography and the development of NAH”. J. Acoust. Soc. Am., 78(4), pp. 1395-1413.

[3] Williams, E. G., Dardy, H. D., and Washburn, K. B., 1987. “Generalized nearfield acoustical holography for cylindrical geometry: Theory and experiment”. J. Acoust. Soc. Am., 81(2), pp. 389-407.

[4] Williams, E. G., 1999. Fourier Acoustics: Sound Radiation and Nearfield Acoustical Holography. Academic Press.

[5] Wu, S. F., 2004. "Hybrid nearfield acoustic holography". J. Acoust. Soc. Am., 115(1), pp. $207-217$.

[6] Pézerat, C., Leclère, Q., Totaro, N., and Pachebat, M., 2009. "Identification of vibration excitations from acoustic measurements using near field acoustic holography and the force analysis technique”. J. Sound Vib., 326, pp. 540-556.

[7] Langrenne, C., Melon, M., and Garcia, A., 2007. "Boundary element method for the acoustic characterization of a machine in bounded noisy environment”. J. Acoust. Soc. Am., 121(5), pp. 2750-2757.

[8] Langrenne, C., Melon, M., and Garcia, A., 2009. "Measurement of confined acoustic sources using near-field acoustic holography”. J. Acoust. Soc. Am., 126(3), pp. 1250-1256.

[9] Ouisse, M., Maxit, L., Cacciolati, C., and Guyader, J.-L., 2005. "Patch transfer functions as a tool to coupla linear acoustic problems". In ASME J. Vib. Acoust., Vol. 127, pp. 458-466.

[10] Ouisse, M., Maxit, L., Cacciolati, C., and Guyader, J.-L., 2004. “Application of a substructuring approach for linear acoustics in automotive context”. In Inter-Noise.

[11] Totaro, N., Andro, B., PÈteul, C., and Guyader, J.-L., 2007. "Extension of the patch transfer functions method (ptf method) to high frequency domain (sub-cavities decomposition)”. In INTER-NOISE 2007.

[12] Totaro, N., and Guyader, J.-L., 2012. "Efficient positioning of absorbing material in complex systems by using the patch transfer function method". J. Sound Vib., 331, pp. 3130-3143.

[13] Vigoureux, D., Totaro, N., and Guyader, J.-L., 2011. “Identification des champs de vitesse, pression et intentié à l'aide de la méthode iPTF”. In 20e Congrès Français de Mécanique.

[14] Hansen, P. C., 1998. Rank-deficient and discrete ill-posed problems. SIAM, Philadelphia, PA.

[15] Leclère, Q., 2009. “Acoustic imaging using under-determined inverse approaches: Frequency limitations and optimal regularization”. J. Sound Vib., 321, pp. 605-619.

[16] Crocker, M. J., 2007. Handbook of noise and vibration control. John Wiley and Sons, United States of America.

[17] Williams, E. G., 1998. “Supersonic acoustic intensity on planar sources”. J. Acoust. Soc. Am., 104(5), pp. 2845-2850.

[18] Fernandez-Grande, E., Jacobsen, F., and Leclère, Q., 2012. "Direct formulation of the supersonic acoustic intensity in space domain". J. Acoust. Soc. Am., 131(1), pp. 186-193.

[19] Corrêa-Junior, C. A., and Tenenbaum, R. A., 2013. "Useful intensity: A technique to identify radiating regions on arbitrarily shaped surfaces". J. Sound Vib., 332, pp. 1567-1584.

[20] Pezerat, C., and Guyader, J.-L., 2000. "Force analysis technique: reconstruction of force distribution on plates". Acta Acustica, 86, pp. 322-332.

[21] Pearson, K., 1901. "On line and places of closest fit to systems of points in space”. Phylosophical magazine, 2(6), 
pp. 559-572.

\section{List of Figures}

1 Example of system under study. A vibrating surface $\Sigma$ radiates in an acoustic volume with a rigid surface $\Lambda$

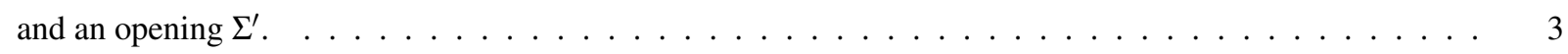

2 System under study: a plate excited by a point force radiating into a semi-infinite acoustic medium. . . . . 8

3 Definition of the virtual surface $\Sigma^{\prime}$ surrounding the rectangular plate. Top view. Heigth $=0.045 \mathrm{~m}$. $\ldots \ldots$

4 Intensity fields at $1900 \mathrm{~Hz}$. (a) Reference field; (b) Identified field. . . . . . . . . . . . . . . . . . . . . 9

5 Space averaged intensity on the surface of the plate as a function of frequency. (a) Comparison of reference and identified intensity; (b) Identification error in $\mathrm{dB} \ldots \ldots \ldots \ldots$. . . . . . . . . . . . 9

6 Influence of the evanescent waves. (a) Penetration depth of evanescent waves of an infinite plate as a function of $\omega / \omega_{c} ;(b)$ reduction factor at the distance $z_{s}$ from the plate as a function of $\omega / \omega_{c} \ldots \ldots \ldots \ldots$

$7 \quad$ Velocity field (a) computed on the surface of the plate (reference), (b) identified by iPTF and radiated velocity fields computed using (c) reference or (d) the identified field at 4 and $20 \mathrm{~cm}$ from the plate at $710 \mathrm{~Hz}$. . . .

8 Identification and measurement meshes used in the study on influence of the identification mesh. (a) Identification mesh $-\lambda_{b} / 2$; (b) Identification mesh $-\lambda_{b} / 4$; (c) Identification mesh - $\lambda_{b} / 6$; (d) Measurement mesh -

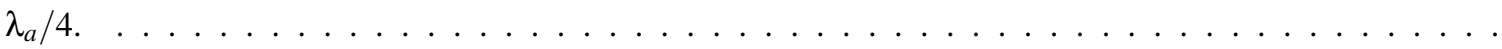

9 Velocity fields identified at $3900 \mathrm{~Hz}$ with the different identification meshes shown in Fig. 8. (a) Reference field; (b) identified field obtained with $\lambda_{b} / 2$ mesh; (c) identified field obtained with $\lambda_{b} / 4$ mesh; (e) identified field obtained with $\lambda_{b} / 6$ mesh $(963$ eigen modes). $\ldots \ldots \ldots \ldots \ldots \ldots$

10 Identification and measurement meshes used in the study on influence of the measurement mesh. (a) Measurement mesh $-\lambda_{a} / 2$; (b) Measurement mesh $-\lambda_{a} / 4$; (c) Measurement mesh - $\lambda_{a} / 6$; (d) Identification mesh

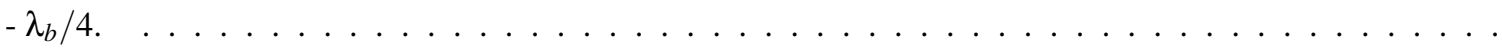

11 Velocity fields identified at $3900 \mathrm{~Hz}$ with the different measurement meshes. (a) Reference field; (b) identified field obtained with $\lambda_{a} / 2$ mesh; (c) identified field obtained with $\lambda_{a} / 4$ mesh; (d) identified field obtained

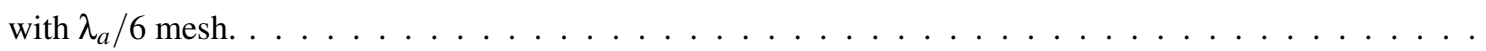

12 Identification of the velocity when gaussian noise is added on (a) measured velocity or (b) measured pressure. 16

13 System under study: an aluminum plate glued to a wood frame and excited by a shaker. (a) Photography of

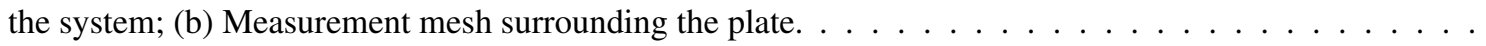

14 Experimental comparison between reference, identified and radiated fields as function of frequency. (a) Sound pressure; $(b)$ mean square velocity $\ldots \ldots \ldots \ldots \ldots \ldots \ldots$

15 Experimental comparison between reference and identified fields at $205 \mathrm{~Hz}$ on the plate surface. (a) real part of the reference pressure field; (b) real part of the reference velocity field; (c) reference active intensity field; (d) real part of the idenfied pressure field; (e) real part of the identified velocity field; (f) identified active

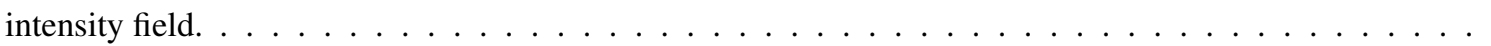


16 Frequency Domain Assurance Criterion between reference and idenfitied velocity fields as a function of frequency. The dashed line indicates the threshold of good correlation $(0.75) \ldots \ldots \ldots \ldots$

\section{List of Tables}

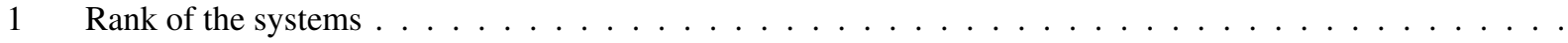

